\title{
Directores Escolares y Padres que Logran Resultados Óptimos: Lecciones Aprendidas de Seis escuelas Norteamericanas que han Implementado Prácticas Inclusivas
}

\section{School Principals and Parents Who Achieve Optimum Results: Lessons Learned from 6 North American Schools that have Implemented Inclusive Practices}

\author{
Grace. L Francis ${ }^{1 *}$ \\ Judith M.S. Gross ${ }^{2}$ \\ Martha Blue-Banning ${ }^{2}$ \\ Shana Haines ${ }^{3}$ \\ Ann P. Turnbull ${ }^{2}$ \\ ${ }^{1}$ George Mason University ${ }^{2}$ University of Kansas ${ }^{3}$ University of Vermont
}

\begin{abstract}
La educación inclusiva aporta numerosos beneficios a los alumnos con y sin discapacidades, incluyendo mejores logros académicos y relaciones con sus pares. Un liderazgo escolar fuerte y comprometido, además de alianzas y relaciones de confianza con las familias son dos características que el Centro SWIFT (Schoolwide Integrated Framework for Transformation Center) de la Universidad de Kansas, utiliza para ayudar a las escuelas a transformarse en establecimientos totalmente inclusivos. Este artículo describe los resultados de 11 grupos focales llevados a cabo con padres de alumnos con y sin discapacidades en seis escuelas norteamericanas, reconocidas por sus prácticas inclusivas. Los resultados de este estudio revelaron varias formas en que los directores y los padres de los alumnos de las escuelas pueden aliarse para contribuir a la vida escolar y a obtener resultados positivos para todas las partes involucradas.
\end{abstract}

Descriptores: Inclusión, Liderazgo, Colaboración, Padres, Dirección escolar.

Inclusive education contributes to numerous benefits for students with and without disabilities, including enhanced academic achievement and relationships with peers. Strong and engaged site leadership and trusting family partnerships are two features that the Schoolwide Integrated Framework for Transformation (SWIFT) Center based at the University of Kansas, uses to help schools transform into fully inclusive schools. This manuscript describes findings from 11 focus groups conducted with parents of students with and without disabilities from six American schools recognized for their inclusive practices. The results of this study revealed several ways in which school principals and parents of students can partner to contribute to the life of schools and positive outcomes for all stakeholders.

Keywords: Inclusion, Leadership, Partnership, Parent, Principalship.

*Contacto: gfranci4@gmu.edu 


\section{Introducción}

Las escuelas inclusivas educan a todos los alumnos en ambientes de aprendizaje que practican la inclusión de todos los niños sobre la base de la equidad, donde cada alumno es valorado como miembro de su escuela del barrio y donde se le proporciona el apoyo necesario para lograr el éxito social y académico (Sailor, 2014). La educación inclusiva tiene múltiples beneficios para los alumnos con o sin discapacidades, incluyendo mayor rendimiento académico y mejores relaciones sociales (Cosier, Causton-Theoharis y Theoharis, 2013; Cushing y Kennedy, 19976; Sermier Dessemontet y Bless, 2013). Las investigaciones indican que las relaciones de confianza entre padres y profesionales y un fuerte y comprometido liderazgo escolar fortalecen las prácticas inclusivas y los resultados de las partes involucradas (Goddard, Tschannen-Moran y Hoy, 2001: Sweetland y Hoy, 2000).

Las relaciones de confianza entre padres y profesionales ocurren cuando los padres y profesionales (profesores y directores) tienen respeto y confianza recíproca, dependen y confían unos de otros, y participan en procesos de toma de decisiones compartidas (Haines, McCart y Turnbull, 2013). La colaboración entre padres y profesionales se traduce en múltiples beneficios para los alumnos, en un mejor rendimiento educacional, mejores logros académicos y conductuales, y aumento en la asistencia de los estudiantes (Bryan y Henry, 2012; Giovacco.Johnson, 2009; Goddard et al., 2001: Lawson, 2003; Tschannen-Moran, 2014). Las relaciones de confianza también producen resultados positivos tanto para los educadores, que se traducen en la mejora de la enseñanza (Haines et al., 2013), como para los padres en cuanto a la satisfacción con la escuela de sus hijos, mejores competencias parentales y relaciones sociales, y reducción de niveles de estrés (Blue-Banning, Summers, Frankland, Nelson y Beegle, 2004; Burke y Hoddap, 2014; Haines et al., 2013; Hill y Taylor, 2004).

A pesar de los beneficios anteriormente señalados, la colaboración entre padres y profesionales a menudo no se concreta en la escuela. Esta ausencia de relaciones con frecuencia se debe a ciertas barreras como la desconfianza de los padres hacia los profesionales, a la falta de información acerca de la colaboración y de la inclusión, y las percepciones negativas que tienen los profesionales de la escuela respecto de los padres de alumnos con discapacidad (Blue-Banning et al., 2004; Cooper, Riehl y Hasan, 2010; Cullen, Gregory y Noto, 2010; Hill y Taylor, 2004). Estas barreras a menudo se ven exacerbadas en las escuelas inclusivas cuando los profesores carecen de capacitación sobre cómo construir alianzas entre padres y profesionales, así como en estrategias de enseñanza efectivas para dar respuesta a la diversidad del alumnado (e.g. diferenciación, co-enseñanza, diseño universal para el aprendizaje, (Cullen et al, 2010). Adicionalmente, muchos padres temen que sus hijos no contarán con el apoyo necesario en ambientes inclusivos o que experimentarán acoso físico o psicológico en la escuela (TASH, 2012). La confianza y un fuerte liderazgo escolar pueden mitigar muchos de estos obstáculos y facilitar las relaciones de colaboración entre padres y profesionales en las escuelas inclusivas (Sandhill, 2010; Waldron y McLeskey, 2010).

La confianza promueve la cooperación y la comunicación (Baier, 1986), mientras que la desconfianza conduce a la ansiedad (Tschannen-Moran, 2014), a la decepción y al control de los intentos (Govier ,1997). La confianza es esencial para el progreso de la escuela y el bienestar de los alumnos, especialmente cuando una escuela pone en marcha 
transformaciones o prácticas innovadoras como la inclusión. Las acciones de los líderes administrativos (e.g., establecer comunicaciones positivas, dar seguimiento a los compromisos, crear políticas inclusivas) influyen en el clima de confianza en el personal de la escuela, así como entre los profesionales y los padres (Ainscow y Sandhill, 2010; Auerbach, 2010; Tschannen-Mor5an, 2001, 2014). Además, los líderes que involucran al personal en acciones de desarrollo profesional relacionado con la asociación con los padres, que crean expectativas para la formación de alianzas y oportunidades para la generación de interacciones positivas, aumentan las probabilidades de que se produzcan relaciones de confianza entre padres y profesionales (Tschannen-Moran, 2014). Un fuerte y comprometido liderazgo escolar y relaciones de confianza con las familias son dos características que el Centro SWIFT utiliza para ayudar a las escuelas a crear escuelas efectivas e inclusivas.

\section{El Centro SWIFT}

El Centro SWIFT (Schoolwide Integrated Framework for Transformation Center), es un centro de asistencia técnica norteamericano con base en la Universidad de Kansas que crea capacidad en las escuelas para proporcionar apoyo académico y conductual con el fin de mejorar el rendimiento académico de todos alumnos entre las edades de 5 a 14 años de edad, incluyendo aquellos con importantes necesidades de apoyo, a través de la inclusión basada en la equidad (Sailor, McCart y Satter, 2014). Los esfuerzos de asistencia técnica de SWIFT ayudan a las escuelas a transformarse en 5 dominios: liderazgo administrativo, sistema de apoyo múltiple, marco educacional integrado, participación familiar y comunitaria, y estructura de política y práctica inclusiva. Cada dominio está representado por dos características basadas en evidencia conocida para mejorar los logros de los alumnos (tabla 1). Un fuerte y comprometido liderazgo escolar y relaciones de confianza entre padres y profesionales son características vitales de los dominios más importantes que dan origen a la fundación SWIFT, liderazgo administrativo y participación familiar y comunitaria.

Tabla 1.Dominios y características de SWIFT

\begin{tabular}{|c|c|c|c|c|}
\hline $\begin{array}{c}\text { LIDERAZGO } \\
\text { ADMINISTRATIVO } \\
\text { (DIRECCIÓN } \\
\text { ESCOLAR) }\end{array}$ & $\begin{array}{l}\text { SISTEMA DE } \\
\text { APOYO } \\
\text { MÚLTIPLE }\end{array}$ & $\begin{array}{c}\text { MARCO } \\
\text { EDUCACIONAL } \\
\text { INTEGRADO }\end{array}$ & $\begin{array}{c}\text { PARTICIPACIÓN } \\
\text { FAMILIAR Y } \\
\text { COMUNITARIA }\end{array}$ & $\begin{array}{c}\text { ESTRUCTURA DE } \\
\text { POLÍTICAS Y } \\
\text { PRÁCTICAS } \\
\text { INCLUSIVAS }\end{array}$ \\
\hline $\begin{array}{l}\text { Fuerte y } \\
\text { comprometido } \\
\text { liderazgo } \\
\text { escolar }\end{array}$ & $\begin{array}{l}\text { Enseñanza } \\
\text { inclusiva } \\
\text { (aprendizajes } \\
\text { académicos) }\end{array}$ & $\begin{array}{l}\text { Estructura } \\
\text { organizacional } \\
\text { totalmente } \\
\text { integrada }\end{array}$ & $\begin{array}{l}\text { Relaciones de } \\
\text { confianza y } \\
\text { colaboración } \\
\text { con la familia }\end{array}$ & $\begin{array}{l}\text { Estrecha relación } \\
\text { entre las } \\
\text { autoridades } \\
\text { locales y la } \\
\text { escuela }\end{array}$ \\
\hline $\begin{array}{l}\text { Sólido sistema de } \\
\text { apoyo educativo }\end{array}$ & $\begin{array}{l}\text { Enseñanza de } \\
\text { actitudes y } \\
\text { conductas } \\
\text { inclusivas }\end{array}$ & $\begin{array}{l}\text { Sólida y positiva } \\
\text { cultura escolar }\end{array}$ & $\begin{array}{l}\text { Relaciones de } \\
\text { confianza y } \\
\text { colaboración } \\
\text { con la } \\
\text { comunidad }\end{array}$ & $\begin{array}{l}\text { Marco de } \\
\text { políticas } \\
\text { autoridades } \\
\text { educacionales } \\
\text { locales }\end{array}$ \\
\hline
\end{tabular}

Fuente: Elaboración propia.

El Centro SWIFT afirma que el fuerte y comprometido liderazgo escolar se produce cuando los directores de escuelas y equipos de liderazgo implementan prácticas inclusivas sostenibles y garantizan procesos de enseñanza, aprendizaje y toma de decisiones compartidas (consultar www.swiftschools.org). El Centro define las 
relaciones de confianza entre familias y profesionales como aquellas que contribuyen a logros de rendimiento escolar positivos y que ocurren cuando a) los miembros de la familia y el personal de la escuela tienen relaciones de mutuo respeto y beneficio, compartiendo la responsabilidad con el aprendizaje de los alumnos; b) los miembros de la familia tienen opciones de involucrarse de manera significativa en la educación de sus hijos y en la vida de la escuela; y c) la escuela responde a los intereses y a la participación de la familia con sensibilidad cultural.

Este artículo examina la intersección de estas características de SWIFT describiendo las perspectivas de los padres de los alumnos con y sin discapacidades que asisten a 6 escuelas seleccionadas por el centro SWIFT en razón de sus prácticas inclusivas. Específicamente, a través de esta indagación se busca responder a dos preguntas: a) ¿Qué estrategias o métodos emplean los directores para crear una cultura escolar inclusiva? y b) ¿cuáles son las implicancias de las acciones de los directores y de la cultura escolar respecto de las relaciones de confianza entre padres y profesionales?

\section{Metodología}

Los investigadores del Centro SWIFT desarrollaron una investigación basada en la indagación apreciativa en 6 escuelas localizadas tanto en comunidades rurales como urbanas para informar sobre los esfuerzos de asistencia técnica del Centro SWIFT (Shogren, McCart, Lyon y Sailor, 2015). Miembros del Consorcio Nacional de Liderazgo del Centro SWIFT (un grupo de líderes e investigadores educacionales de toda la nación) propusieron 37 escuelas y de las cuales fueron seleccionadas 5 escuelas primarias y una escuela media (nivel educacional entre la escuela primaria y la escuela secundaria y que abarca los grados 6,7 y 8) para estudiar buenas prácticas relacionadas con una o más características del Centro SWIFT (incluyendo un fuerte y comprometido liderazgo escolar y relaciones de confianza entre padres y profesionales). Estas escuelas se encuentran ubicadas en 5 estados norteamericanos (California, Florida, Massachusetts, Missouri y Wisconsin).

\subsection{Participantes}

Los investigadores del Centro SWIFT que forman parte del equipo de Participación de la Familia y la Comunidad, llevaron a cabo once grupos focales con padres de estudiantes que asistían a las escuelas seleccionadas. Seis de los grupos consistían en padres de alumnos con discapacidades (p.ej., trastornos del aprendizaje, alteraciones del habla y del lenguaje, discapacidades físicas, intelectuales y del desarrollo) y 5 incluían a padres considerados por las autoridades de las escuelas como "líderes" (p.ej., padres involucrados en las actividades a nivel del aula, la escuela y del distrito). Los participantes eran predominantemente mujeres (49 madres y nueve padres), y el tamaño de los grupos fluctuó entre 4 a 12 participantes. En un grupo se incluyó a un intérprete para un padre cuya primera lengua era el español y no el inglés.

\subsection{Recolección de datos}

Los once grupos focales fueron conducidos por dos investigadores del equipo de Participación de la Familia y la Comunidad del Centro SWIFT y duraron en promedio 1 hora 30 minutos. Uno de los investigadores actúo como facilitador principal realizando las siguientes tareas: a) obtener consentimiento informado de todos los participantes, b) 
explicar la naturaleza de la investigación e iniciar las presentaciones, y c) aplicar un protocolo con preguntas específicas y otras complementarias de sondeo con el fin de guiar las discusiones grupales. Este protocolo contemplaba preguntas relacionadas con la forma en que los profesionales de las escuelas creaban relaciones de confianza con las familias (p. ej., "Cuénteme acerca de su relación con el profesor de su hijo"). El segundo investigador tomó notas de campo, se encargó de grabar las discusiones y de controlar el tiempo. Todas las grabaciones de audio fueron transcritas de manera de contar datos abundantes y confiables para iniciar el análisis.

\subsection{Análisis de datos}

Para el análisis de la información los investigadores utilizaron el software cualitativo Dedoose (2013) que les ayudó a la organización de los datos transcritos y a la construcción de las categorías. Para iniciar el análisis, dos miembros del equipo investigador realizaron una codificación abierta de las mismas dos transcripciones a objeto de determinar categorías generales para ser utilizadas en el desarrollo de un libro de códigos inicial (Cresswell, 2009). Una vez que se elaboró el libro de códigos, procedieron a utilizarlo para codificar transcripciones adicionales, realizando reuniones periódicas para analizar las transcripciones, revisar las categorías y en caso necesario agregar subcategorías con el fin de esclarecer los códigos y producir una versión final del libro de códigos.

A continuación, dos investigadores del equipo que utilizaron el libro final de códigos participaron en la codificación axial de todas las transcripciones. A través de las codificaciones axiales, los investigadores analizaron los datos de los grupos focales, utilizando el libro final de códigos, determinando tanto la pertinencia de los códigos como la conceptualización de las categorías en relación recíproca (Charmaz, 2005). En esta etapa, se identifica el fenómeno o tema central y los investigadores regresan a los datos para comprender en profundidad las categorías específicas que explican o se relacionan con el fenómeno o tema central que emergió (Cresswell, 2009)

\section{Resultados}

Este estudio cualitativo dio como resultado numerosos hallazgos relacionados con factores que facilitan relaciones de confianza entre padres y profesionales, incluyendo: a) cultura escolar de inclusión; b) liderazgo administrativo; c) atributos de relaciones positivas; d) oportunidades para la participación de la familia; y d) resultados positivos para todos los alumnos (Francis, Blue-Banning, Turnbull, Haines, Gross y Hill, en prensa). Para efectos de este artículo, nos centramos en las percepciones de los participantes sobre las formas en que el liderazgo escolar, específicamente de los directores, crearon una cultura escolar donde todas las familias se sentían incluidas. Nuestro análisis indicó que aunque los enfoques individuales diferían, los directores utilizaron métodos similares para crear culturas escolares inclusivas, las que, a su vez, crearon oportunidades y motivación para que los padres participaran en asociación con los profesionales. En la próxima sección se analizan a) los roles de los directores al crear una cultura escolar inclusiva; b) experiencias transformadoras de los padres; c) los padres como colaboradores en escuelas inclusivas. 


\subsection{Roles de los directores en la creación de una cultura inclusiva}

Los participantes de todos los grupos utilizaron numerosos términos positivos para describir la cultura escolar, tales como: "solidario", "comunidad", "valorado", "colaborativo", "acogedor", "familia”, "respeto", "maravilloso", "genuino", “divertido” y "amistoso". Si bien los participantes recordaron con gusto muchas experiencias positivas vividas con los profesores y personal de la escuela, destinaron un tiempo considerable a analizar la importancia del director/a en la "preparación del terreno" para que prosperara la relación entre padres y profesionales. Todos los participantes manifestaron que conocían y confiaban en el director/a de la escuela de sus hijos/as. Los participantes también describieron sentir una profunda conexión con la escuela, y la sensación de que todos "tenían un sentido de pertenencia".

Por su parte, los directores de todas las escuelas utilizaron numerosos métodos para crear ese sentido de pertenencia, promoviendo una cultura inclusiva dentro de las escuelas que se refleja en: a) crear una presencia acogedora en la escuela; b) apoyar eventos escolares; c) identificar y abordar necesidades de los alumnos y las familias), d) mantener altas expectativas y estándares para el personal de la escuela, y e) distribuir el liderazgo entre los profesionales de la escuela y los padres.

\subsubsection{Creación de una presencia acogedora en la escuela}

Los participantes de una escuela describieron cuan accesible y "abordable" era el director de la escuela, destacando la manera "relajada" como se comunicaba y vestía (e.g. el director de escuela comúnmente usaba pantalones de color caqui y sandalias en lugar de traje y corbata). Los participantes de la mayoría de las escuelas aludieron a la importancia de que los directores conozcan los nombres de los alumnos y padres y de que estén "en los pasillos" (en vez de estar en la oficina), facilitando la iniciación de conversaciones amistosas e informales. Muchos padres indicaron sentirse inicialmente sorprendidos por la calidez y el carácter sociable de los directores. Los participantes contrastaron sus experiencias con los directores de las actuales escuelas de sus hijos con experiencias negativas en otras escuelas (e.g. los padres sentían que no se les respetaba, que a la escuela no le importaban sus hijos), como asimismo con sus propias experiencias como alumnos cuando el director era considerado como "una persona encargada de hacer cumplir la disciplina” y a quien sólo veían cuando se involucraban en problemas.

\subsubsection{Apoyo a eventos escolares}

Los participantes de todas las escuelas también resaltaron los "maravillosos" eventos patrocinados y actividades que los directores apoyaban para que asistieran los alumnos con sus familias. Ejemplos mencionados de este tipo de actividades fueron: desfiles, bailes, actos multitudinarios, carreras familiares benéficas, eventos reminiscentes de la época escolar, y conciertos. Los padres de una escuela describieron cómo el director se asoció con las familias y la comunidad para reactivar un evento escolar con el fin de apoyar la alfabetización.

Teníamos aquí caballos como de miniatura... $\Upsilon$ los caballos usaban disfraces (relacionados con libros)... y ellos (el personal) leían una historia y los niños llegaban a ser parte de la historia con cada caballo. $\Upsilon$ ellos solían llamar a algunos niños y luego todos se juntaban y le hacían cariño a los caballos. Uno de ellos, una persona o un grupo pequeño de personas no pudo lograrlo. Eso fue absolutamente una participación comunitaria, allí había también participación parental. 
Los directores no sólo apoyaban estas actividades ayudando con la planificación, organización y la recolección de fondos, sino que también asistían a todos los eventos, conversando de manera entusiasta con las familias, tomando fotos, y a menudo sorprendiéndolos con algunas bromas divertidas. Por ejemplo, para el primer día de clases después del receso de verano el personal de una escuela se vistió con disfraces temáticos para dar la bienvenida a los alumnos y sus familias.

\subsubsection{Identificación y manejo de la necesidades de alumnos y padres}

Los participantes también recordaron formas en que los directores identificaron y abordaron las necesidades de las familias (p.ej., las necesidades de los padres, estudiantes, hermanos), como afrontar pronta y efectivamente el acoso escolar físico o psicológico; proveer los recursos necesarios de transporte hacia la escuela; garantizar que esta fuese accesible a los alumnos con discapacidades y/o con diferentes realidades lingüísticas y culturales. Un director estaba muy comprometido en colaborar con los padres y alumnos (p.ej., piscina comunitaria). Otro director de una escuela ubicada en una comunidad rural, lideró el esfuerzo para recolectar alimentos, mochilas, ropa, abrigos, y otros elementos relacionados con necesidades básicas para los alumnos y sus familias. Los alumnos podían tener acceso a estos materiales a lo largo de todo el año, según fuese necesario.

3.1.4. Mantenimiento de altas expectativas y estándares para el personal administrativo de la escuela

Los participantes también analizaron la importancia de que los directores mantengan altas expectativas y estándares para el personal de la escuela, incluyendo competencias para la enseñanza de todos los estudiantes (incluidos aquellos con discapacidades o de diversas realidades lingüísticas o culturales) como también expectativas en torno a la colaboración con las familias y el involucramiento de los padres en la educación de los estudiantes. Los directores en todas las escuelas demostraron expectativas en diversas formas, tales como: comunicarse a través de correos electrónicos y boletines informativos, contratando a "la gente indicada" y "dejando ir a aquellos que no funcionaban bien" o "que no deseaban estar aquí o que no deberían estar aquí". Estas acciones eran valoradas por los participantes porque les permitía "ver que en realidad habían cambios" cuando un profesor no cumplía con las expectativas establecidas. Por otra parte, los directores celebraban a los padres y profesionales de la escuela por "estar presentes" y reconocían los esfuerzos y logros a través de cartas de reconocimiento enviadas a los hogares de las familias.

Aunque los métodos variaban, los directores de todas las escuelas de manera consistente mostraron los estándares y conductas que ellos esperaban tanto de parte de los profesores como de los padres. Todos los directores mantenían una comunicación frecuente y honesta con el personal de la escuela, padres, y alumnos. También solicitaban ayuda cuando la requerían y compartían los éxitos como también los desafíos que enfrentaban. Consistentemente, los directores tomaron decisiones centradas en los alumnos, orientadas a mejorar su rendimiento y calidad de vida.

\subsubsection{Distribución del liderazgo entre profesionales de la escuela y padres}

Asimismo los directores abogaban por un liderazgo compartido entre los profesionales de la escuela y los padres. Cada escuela tenía un equipo de formación en liderazgo facilitado por el director, compuesto por profesores, personal de la escuela, padres y otras partes interesadas (p. ej., servicios relacionados con la escuela como terapeutas 
ocupacionales). Estos equipos se reunían para analizar información relevante de la escuela y a través del enfoque de la lluvia de ideas consolidar las fortalezas y abordar áreas de preocupación (p. ej., logro académico, asistencia, satisfacción profesor/padre) para mejorar el rendimiento estudiantil.

Los directores también extendieron las oportunidades de liderazgo a los padres involucrándolos en los comités escolares, instancias en la que se toman decisiones relacionadas con la asignación de fondos, contratación de nuevos profesores, selección de nuevos currículos, y revisión de políticas escolares. Examinaron y sostuvieron audiencias con los padres para obtener información de las personas que no podían o preferían no unirse formalmente a un comité. En algunas escuelas, los directores junto con los profesionales y padres a asistieron a reuniones de la junta escolar y a otras reuniones de carácter legislativo a nivel distrital y estatal para abogar por políticas que consideraban beneficiarían a los alumnos. Finalmente, los directores se mostraban receptivos a las sugerencias e ideas propuestas por los padres. Un participante describió la forma en que el director de la escuela de su hijo reaccionó a las ideas expresadas por los padres, "Sí, sí, sí, si ustedes pueden lograr que funcione, hagámoslo"...(el director) "ayuda a orientar en lo que más necesita la escuela, pero nunca se niega a la posibilidad de escuchar". Un director de otra escuela apoyó las iniciativas propuestas por los padres, la comunidad y los alumnos conectándolos con los recursos apropiados y ayudándolos a explorar las políticas asociadas con la escuela, el distrito, o la comunidad. Por ejemplo, una escuela urbana tenía un horno para pizza y un jardín comunitario que estaban disponibles para toda la comunidad escolar. Todas estas ideas fueron propuestas por los padres o los estudiantes y apoyadas por el director. Además de los múltiples métodos que los directores utilizaron para crear un clima escolar inclusivo, los participantes enfatizaron fuertemente el impacto positivo que este cálido y acogedor clima escolar tenía sobre su participación y compromiso con la escuela de sus hijos.

\subsection{Experiencias transformadoras de los padres}

En todos los grupos, un número importante de padres compararon sus experiencias previas en ambientes escolares no-inclusivos y carentes de apoyo, con las experiencias vividas en la escuela actual a la que asisten sus hijos. En las escuelas anteriores, los padres recordaron experiencias incómodas, "aterradoras", e incluso beligerantes con directores de escuelas no-inclusivas que habían terminado en "situaciones formales de mediación con la participación de un abogado". Un padre describió sus intentos por colaborar con el director y el personal de la anterior escuela de su hijo como una "súper batalla". Este padre recordó haber pasado horas antes de reunirse con los profesionales de la escuela, revisando leyes estatales y federales con el fin de preparar los "argumentos" que utilizaría para abogar por "exactamente lo que los niños necesitan frente a lo que la escuela puede ahorrar en dinero".

Estos mismos padres describieron estar "sorprendidos" ante las diferencias que observan respecto de la cultura escolar y las relaciones que se dan entre padres y profesionales en la actual escuela de sus hijos, describiendo cómo las actuaciones de los directores desarmaron y distendieron a los padres quienes, en escuelas anteriores, sentían que tenían que prepararse para "batallar" contra la dirección escolar para velar por una educación adecuada para sus hijos. Asimismo, señalaron la diferencia en "espíritu", "cultura", y "filosofía" que se apreciaba en las reuniones del Programa de Educación Individualizada (IEP), donde los padres eran tratados "como expertos" en sus hijos en 
vez de ser una carga para el profesor. Estos padres también analizaron como, en contraste con la naturaleza de las reuniones anteriores principalmente "orientada a metas", la naturaleza y contenido de las reuniones en las escuelas actuales "excedieron...(lo que se requiere según) la ley federal". Percibieron a los directores de las escuelas actuales como campeones para sus hijos, en vez de adversarios. Aunque los padres con experiencias previas negativas reconocieron dificultades iniciales "para salir de una situación de confrontación", las acciones de los directores y la consecuente cultura escolar inclusiva consolidaron relaciones de confianza, las que a su vez, alentaron y motivaron a los padres a aliarse con los profesionales de la escuela.

\subsection{Los padres como colaboradores en escuelas inclusivas}

A medida que los directores creaban una cultura escolar inclusiva y generaban oportunidades para establecer relaciones de confianza entre padres y profesionales, los padres se sintieron correspondidos al colaborar con el equipo y contribuir a la educación de sus hijos como también a la vida de la escuela. Los padres actuaron como colaboradores en: a) la toma de decisiones educacionales; b) comunicación entre escuelahogar; c) liderazgo escolar; y d) apoyo a la participación de los padres en las escuelas.

\subsubsection{Colaboradores en la toma de decisiones educacionales}

Los participantes informaron sentirse "empoderados" y capaces de hacer cambios en las escuelas de sus hijos. Una madre analizó la forma en que ella contribuía a la educación de su hijo al ofrecer al personal de la escuela "diferentes estrategias que uso (en casa)... "que el personal pudo incorporar a la escuela". La cultura de "apertura· y "aceptación" que los directores crearon en las escuelas hicieron que los padres se sintieran seguros al formular preguntas, ofrecer sugerencias, dar a conocer sus opiniones (incluyendo objeciones), y participar en la toma de decisiones con los profesores sin sentirse como el "tipo malo". Por ejemplo, una madre describió una conversación que tuvo con el profesor acerca de las razones por las cuales ella no deseaba que su hijo completara sus tareas en la noche: "Me paso dos horas... con mi hijo por la noche. No voy a ayudarlo si discuto con él y como sabe (los profesores) se encargan de ello, y no es un problema. Ellos me escuchan de igual modo como yo los escucho".

\subsubsection{Colaboradores en la comunicación escuela-hogar}

Los padres se comunicaban regularmente con el personal de la escuela acerca de sus hijos para darles una "mano" y ayudar a garantizar el éxito de los alumnos en la escuela. Por ejemplo, describieron informarles a los profesores si sus hijos no conciliaban bien el sueño la noche anterior o si un miembro de la familia que prestaba apoyo estaba fuera de la ciudad. Los métodos para comunicarse variaban entre las escuelas y entre los padres y el personal de una misma escuela. Destacaron que una comunicación efectiva era frecuente, consistente, honesta, informal, y de cierta manera, parecía confortable tanto para los padres como para los profesionales. Por ejemplo, un participante cuya primera lengua no era el inglés prefirió escribir en una revista escuela-hogar en lugar de tener una conversación telefónica o en persona debido a que "es muy fácil entender al leer en inglés, por lo tanto no tengo que lidiar con (inglés hablado) No entiendo cuando hablo por teléfono." 


\title{
3.3.3. Colaboradores en liderazgo escolar
}

Los participantes describieron la forma en que los padres en la escuela sacaban provecho de la buena disposición del director para distribuir el liderazgo y apoyar nuevas iniciativas, "puedes llevarle (al director) ideas acerca de programas, cosas que desees hacer...", reafirmaron cómo "los padres juegan un rol activo en la configuración de los programas (escolares) al compartir ideas, proponer sugerencias y actuar en comités y en grupos de liderazgo. Un padre aludió a la cultura "acogedora y efectiva" de un comité de liderazgo escolar en el que participan:

\begin{abstract}
No tengo la impresión que otros comités escolares o incluso consejos de padres estén tan orientados hacia los padres como yo diría lo son nuestras reuniones y pienso que el rol que los padres desempeñan en esta escuela es mucho más significativo que en otras escuelas donde tengo la sensación que algunas de nuestras otras escuelas son mucho más autoritarias por naturaleza, ya que es el director quien determina lo que va a pasar o lo que se va a analizar y supongo que esos comités no son tan influyentes.
\end{abstract}

Varios participantes que asumieron roles de liderazgo analizaron las formas que usaron para llegar a las familias que no podían o no elegían asumir roles activos en la escuela, de modo de garantizar que se sintiesen vinculados al proceso y que sus opiniones estuviesen representadas. Algunos de ellos compartieron estrategias orientadas a que las escuelas y los padres lograran una participación activa de un mayor número de líderes parentales, "No cree grandes expectativas como necesito cien padres"; "Comience con el grupo principal. Si son sólo cinco o seis personas, empiece con ese grupo y luego trate de partir de allí...”

\subsubsection{Colaboradores en apoyar la participación de los padres en la escuela}

Por otra parte, los participantes analizaron las formas en que facilitaron o apoyaron a otros padres para promover su participación en la escuela. Ejemplos de proporcionar apoyo para la participación de los padres incluyen: a) ayudar a cuidar de los niños en actividades escolares de modo que los padres con muchos hijos puedan asistir; b) proveer cuidado de los niños en actividades extra escolares para apoyar a las familias (ej. Ayudar a que los padres puedan hacer compras navideñas); c) comunicar a los padres acerca de nuevas reuniones y eventos a través de llanadas telefónicas y correos electrónicos; d) realizar video grabaciones de las reuniones y talleres para padres sobre el proceso del Programa de Educación Individualizada ((IEP), derechos de educación especial y luego difundir los videos a través de Youtube; e) crear grupos de apoyo a los padres, y f) facilitar el acceso a clubes de lectura para padres.

Los padres de varias escuelas describieron la presencia de un "acogedor equipo de bienvenida" diseñado para recibir a la nuevas familias que se integran a la escuela y la comunidad. Los padres líderes de estos equipos combinaron familias con mayor antigüedad en la escuela con familias recién llegadas a la escuela con referencia a la edad de los alumnos. Ellos incentivaron a las familias más antiguas a "tratar de llegar" a las nuevas familias e invitarlas a reunirse para almorzar o tomar café y programar fechas para obras teatrales estudiantiles. Los padres de las escuelas rurales informaron haber tomado medidas extras para ir a conocer o pasar a buscar a nuevas familias que vivían en las afueras de la ciudad, o que no tenían transporte, Internet, o teléfono para garantizar que no fuesen excluidos. 


\section{Discusión}

Un fuerte y comprometido liderazgo escolar y relaciones de confianza entre padres y profesionales de la escuela mejoran las prácticas inclusivas y los resultados de todos los involucrados (Goddard et al., 20001; Sweetland y Hoy, 2000). Este estudio investigó las perspectivas de los padres de los niños con y sin discapacidades en 6 escuelas de los Estados Unidos, reconocidas por implementar prácticas inclusivas, relativas a las formas en que el liderazgo escolar, específicamente los directores escolares, crean una cultura inclusiva y la forma en que esta influye en relaciones de confianza entre padres y profesionales. La tabla 2 resume las formas en que los directores y los padres establecieron relaciones de confianza y colaboraron para contribuir a la vida de la escuela y a resultados positivos para los actores involucrados.

Tabla 2. Actividades de colaboración entre directores de escuela y padres

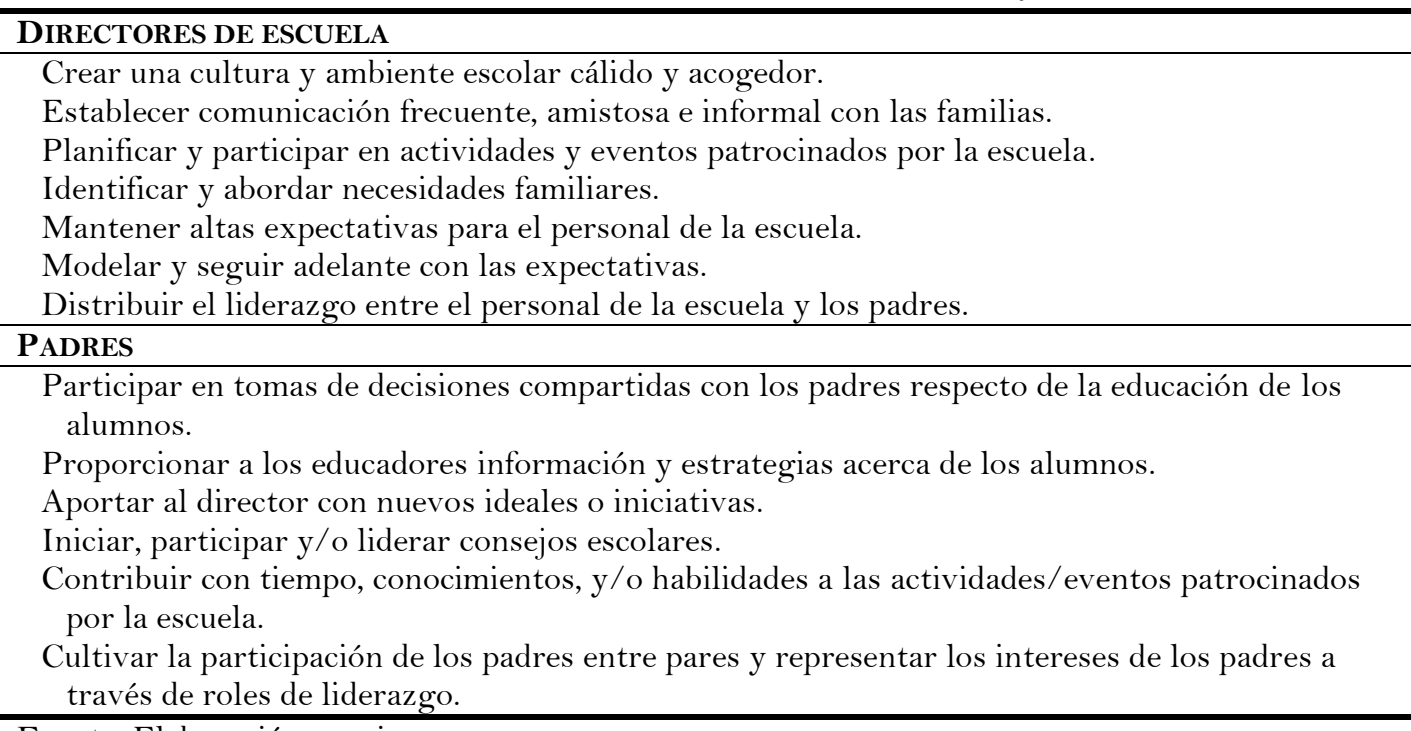

Fuente: Elaboración propia.

Esta investigación realizada en escuelas de distintos estados de los Estados Unidos, seleccionadas por el Centro SWIFT por implementar prácticas ejemplares relacionadas con una o más características de SWIFT (tabla 1), nos permitió indagar sobre cómo influía el fuerte y comprometido liderazgo escolar en la cultura escolar y en las relaciones de confianza entre padres y profesionales. Nuestros hallazgos indican que las acciones de los directores y de los padres que se presentan en la tabla 1, contribuyeron a generar resultados positivos en los distintos actores involucrados y al progreso de la escuela. Cuando escuchamos el término "escuelas inclusivas", a menudo pensamos en alumnos con y $\sin$ discapacidades que aprenden juntos. Sin embargo, nuestras conclusiones indican que en las escuelas que implementan prácticas inclusivas, la inclusión se extiende a los profesionales de la escuela y a las familias de los alumnos/as. Este hallazgo es relevante considerando la influencia que la cultura de la escuela tiene en la excelencia escolar y en los resultados positivos de los actores implicados, incluyendo la satisfacción de los profesores, los resultados de los alumnos, y la participación de los padres (McKinney, Labat y Labat, 2015).

Los resultados de nuestra investigación reflejan los aportes de la literatura sobre las mejores prácticas que los directores pueden poner en marcha para desarrollar una sólida 
cultura escolar y asociación entre padres y profesionales. Por ejemplo, McKinney y colegas (2015) observaron que los líderes transformadores son fuente de inspiración para otros, identifican y satisfacen necesidades y carencias, y distribuyen el liderazgo. Nuestros resultados también confirman el valor de estrategias destacadas en la literatura de data anterior, como visitar salas de clase, comunicar altas expectativas, saludar a padres y alumnos, crear tradiciones escolares y comunicarse con respeto (Elmore, 2000; Robinson. Lloyd y Rowe, 2008). Las investigaciones sobre los directores efectivos destacan la importancia de que los directores establezcan una clara misión (Habegger, 2008; Leithwood, Patten y Jantzi, 2010; Peterson y Deal, 2002). Los directores de nuestro estudio transmitieron una misión de inclusión y resultados positivos de los alumnos. Esta misión estableció los cimientos para una cultura de inclusión en el conjunto de la escuela. Finalmente, la investigación confirmó que los directores que facilitan y aprovechan el capital social, colaborando con los padres, mejoran los resultados de los involucrados (Eptein, Galindo y Sheldon, 2011; Gurr, Drysdale y Mulford, 2005).

Además de confirmar las conclusiones de las investigaciones sobre las mejores prácticas conducentes a una fuerte y sólida cultura escolar y alianzas entre padres y profesionales, esta indagación contribuye a este campo de estudio en numerosas y novedosas maneras. Por ejemplo, nuestro estudio ocurre en 6 escuelas inclusivas de distintos estados de los Estados Unidos e incluye las opiniones de padres que tienen hijos con y sin discapacidades y que reportan variados niveles de liderazgo y participación escolar. Adicionalmente, este estudio resalta cómo las acciones de los directores y colaboradores se entrelazan y se traducen en relaciones de confianza.

Este estudio destaca cómo los directores crearon culturas escolares acogedoras e inclusivas, las que -a su vez- incentivaron la asociación y trabajo colaborativo entre padres y profesionales. Los resultados de este estudio también revelan un notable entrelazamiento de las características SWIFT de un fuerte y comprometido liderazgo y relaciones de confianza entre familias y profesionales de la escuela; tanto los directores como los padres contribuyeron a generar relaciones y resultados positivos para la escuela, las familias y los alumnos. Las actividades en que estos participaron fueron recíprocas, en términos de que tanto los directores como los padres intercambiaron ideas y acciones valoradas por ambos. Como resultado, ambas partes no sólo aportaron sino que también salieron beneficiadas a partir de las contribuciones y actividades desarrolladas. Todas las contribuciones fueron acogidas y valoradas por las distintas partes. El rol o contribución de una persona nunca fue percibido como más importante que el de otra (esto se observó incluso en el caso de padres que no asumieron roles de liderazgo). Este sentido fundamental de pertenencia y equidad se refleja en una cultura escolar inclusiva que valora todos los aportes y a todas las personas.

Los resultados de este estudio también indican que una comunicación positiva, frecuente y honesta entre los integrantes de la comunidad escolar, está en la esencia de las relaciones basadas en la confianza. La figura 1 muestra cómo los resultados de este estudio resaltan el entrelazamiento de las características de SWIFT referente a un fuerte y comprometido liderazgo y relaciones de confianza entre padres y profesionales, y cómo éstas pueden contribuir a dar vida a las escuelas inclusivas. 


\subsection{Limitaciones}

Existen 3 limitaciones importantes de considerar en este estudio. La primera tiene que ver con el proceso de selección de las escuelas. No contamos con personal que pudiera intencionar la selección de los participantes (Maxwell, 2005) con base a los criterios previamente definidos y proporcionados a las escuelas (ej. características de los líderes, representativos de la demografía de la escuela). Este situación limitó capacidad del equipo investigador de garantizar que los actores considerados en el estudio cumplieran de manera rigurosa los criterios que establecidos. La segunda limitación es que los informantes clave fueron padres de alumnos que asistían a 5 escuelas primarias y una escuela media (nivel intermedio entre la escuela primaria y la secundaria). Ello limitó nuestra comprensión acerca de la perspectiva de los padres de alumnos que están prontos a ingresar a la escuela secundaria. La tercera limitación es que, con el fin de proteger la confidencialidad de los participantes y en cumplimiento con la normativa de nuestra Junta de Revisión Institucional (IRB), es posible reportar una restringida información sobre los participantes y las características demográficas de las escuelas estudiadas. Esta restricción limita la generalización de los resultados.

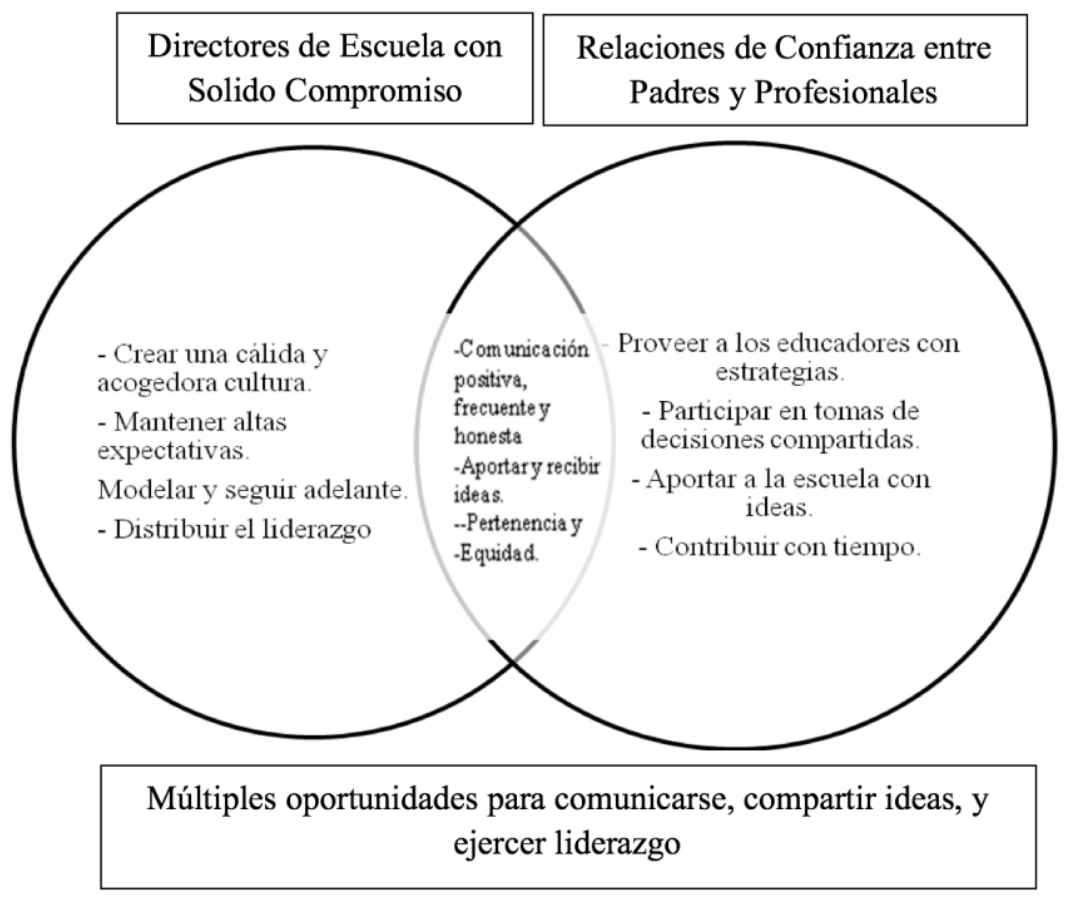

Figura 1. El entrelazamiento de las características de SWIFT de un fuerte y comprometido liderazgo y relaciones de confianza entre familia y profesionales Fuente: Elaboración propia.

\subsection{Investigación futura}

Dada la diversa representación geográfica de las escuelas incluidas en nuestra investigación, los resultados de este estudio pueden ser relevantes para las escuelas en todos los Estados Unidos. Al mismo tiempo, investigadores y actores educativos de otros países pueden considerar cómo los resultados de este estudio se relacionan con las escuelas de sus comunidades locales. No obstante ello, notables beneficios se suman del estudio de Sistemas Escolares que son geográfica, programática y demográficamente 
similares, en tanto aumenta las posibilidades de generalización y la relevancia de los resultados. Por consiguiente, los investigadores internacionales deberían considerar llevar a cabo un estudio similar de desarrollo de conocimientos (ej. identificar escuelas que implementan prácticas inclusivas y ponen énfasis en relaciones de confianza entre padres y profesionales de la escuela) en sus países nativos para:

a) erradicar mitos comunes de inclusión (e.g. las necesidades de los alumnos no serán satisfechas, los alumnos se atrasarán o quedarán rezagados, la inclusión es demasiado costosa)

b) explorar cómo las escuelas abordaron los obstáculos para generar inclusión

c) aprender acerca de las prometedoras prácticas inclusivas, incluyendo un fuerte y comprometido liderazgo escolar y relaciones de confianza entre padres y profesionales de la escuela.

A pesar de la creciente comprensión de los beneficios de la inclusión y un énfasis para que las escuelas la pongan en práctica, los programas universitarios para la formación de profesores, en gran medida, aún forman a los docentes de manera separada en educación general y en educación especial. Esta práctica de formar a los profesores aislados los unos de los otros contribuye a generar profesionales que no están preparados para colaborar y apoyar a todos los alumnos en ambientes inclusivos (Sailor, 2009). Sumado a esto, son escasos los programas de formación docente que incorporan cursos dedicados al desarrollo de competencias para la construcción de relaciones de colaboración con la familia y entre los distintos miembros de la comunidad a fin de proporcionar suficiente apoyo integral a los alumnos como también para el desarrollo de escuelas y comunidades sólidas.

Como se observó en el apartado referido a las limitaciones, nuestro estudio estuvo fuertemente orientado hacia escuelas primarias. Investigaciones futuras podrían indagar sobre las prácticas inclusivas en escuelas medias y secundarias (alumnos cuyas edades fluctúan entre 13 a 18 años). Aunque es probable que el rendimiento escolar en los niveles elementales (Goddard et al., 2001), niveles medios (Sweetland y Hoy, 2000), y niveles secundarios (Hoy y Tarter, 1997), sea más alto en las escuelas en que existe una relación de confianza y colaboración, que en aquellas donde la capacidad de colaborar y la confianza son escasas. De todas formas, la inclusión y las alianzas de colaboración entre padres y profesionales tienden a disminuir a medida que sube la edad de los alumnos (Martinez, Conroy y Cerrero, 2012).

Por último, un estudio longitudinal de estudiantes que asisten a escuelas primarias que implementan prácticas inclusivas (como los alumnos de las escuelas involucradas en este estudio) y de sus padres, para examinar sus experiencias educativas en nuevos ambientes fortalecería nuestra comprensión de los beneficios de la inclusión. Un estudio de esa naturaleza sería especialmente informativo si examinase las formas en que los padres y los alumnos abordan las barreras que dificultan la inclusión, considerando las relaciones de confianza y la colaboración entre las familias y profesionales de la escuela.

\section{A modo de conclusión}

A medida que nuestra cultura se desplaza de la expectativa de la segregación como medio para satisfacer las necesidades de alumnos excepcionales hacia una expectativa de 
inclusión en toda la escuela y la comunidad, la investigación acerca del apoyo efectivo a los alumnos con necesidades diversas es esencial para garantizar que la inclusión tenga sentido y éxito en oposición a estar incluido físicamente en la escuela. Las investigaciones han demostrado la importancia de la participación de la familia en el rendimiento y éxito escolar del estudiante. Este estudio adicionalmente contribuye a ese tipo de investigación al focalizarse en ambientes escolares inclusivos y en el rol del director de escuela y los padres para asegurar relaciones positivas de colaboración que faciliten los procesos de inclusión no solamente de los estudiantes, sino de toda la familia en la vida de la escuela.

\section{Agradecimientos}

Los autores redactaron este artículo según regulaciones del Ministerio de Educación de los Estados Unidos con financiamiento $\mathrm{N}^{\circ} \mathrm{H} 325 \mathrm{Y} 120005$ de la Oficina de Programas de Educación Especial, Centro Nacional sobre la Reforma Escolar Inclusiva a nivel de toda la Escuela: Centro SWIFT. Los Responsables del Proyecto OSEP Grace Zamora Durán y Tina Diamond actuaron como tales. Los puntos de vista que aquí se presentan no necesariamente representan las opiniones o políticas del Ministerio de Educación.

\section{Referencias}

Ainscow, M. y Sandhill, A. (2010). Developing inclusive education systems: the role of organisational cultures and leadership. International Journal of Inclusive Education, 14(4), 401-416. doi:10.1080/13603110802504903

Auerbach, S. (2010). Beyond coffee with the principal: Toward leadership for authentic schoolfamily partnerships. Journal of School Leadership, 20, 728-757.

Baier, A. (1986). Trust and antitrust. Ethics, 96(2), 231-260.

Blue-Banning, M., Summers, J. A., Nelson, L. L. y Frankland, C. (2004). Dimensions of family and professional partnerships: constructive guidelines for collaboration. Exceptional Children, 7O(2), 167-184. doi:10.1177/001440290407000203

Bryan, J. y Henry, L. (2012). A model for building school-family-community partnerships: principles and process. Journal of Counseling \& Development, 90, 408-420. doi:10.1002/j.1556-6676.2012.00052.x

Burke, M. M. y Hodapp, R. M. (2014). Relating stress of mothers of children with developmental disabilities to family-school partnerships. Intellectual and Developmental Disabilities, 52, 1323. doi:10.1352/1934-9556-52.1.13

Charmaz, K. (2006). Constructing grounded theory: a practical guide through qualitative analysis. Londres: Sage.

Cooper, C. W., Riehl, C. J. y Hasan, A. L. (2010). Leading and learning with diverse families in schools: critical epistemology amid communities of practice. Journal of School Leadership, 20, $758-788$.

Cosier, M., Causton-Theoharis, J. y Theoharis, G. (2013). Does access matter? Time in general education and achievement for students with disabilities. Remedial and Special Education, 34(6), 323-332. doi:10.1177/0741932513485448

Creswell, J. W. (2013). Research design: qualitative, quantitative, and mixed methods approaches. Thousand Oaks, CA: Sage. 
Cullen, J. P., Gregory, J. L. y Noto, L. A. (2010). The teacher attitudes toward inclusion scale (TATIS) technical report. Carrollton, GA: Eastern Educational Research Association.

Cushing, L. S. y Kennedy, C. H. (1997). Academic effects of providing peer support in general education classrooms on students without disabilities. Journal of Applied Behavior Analysis, 30(1), 139-151. doi:10.1901/jaba.1997.30-139

Elmore, R. (2000). Building a new structure for school leadership. Washington, DC: Albert Shanker Institute.

Epstein, J., Galindo, C. y Sheldon, S. (2011). Levels of leadership: effects of district and school leaders on the quality of school programs of family and community involvement. Education Administration Quarterly, 47(3) 462- 495. doi:10.1177/0013161x10396929

Francis, G. L., Blue-Banning, M., Turnbull, A. P, Haines, S. J., Gross, J. M. S. y Hill, C. (in press). The culture of community in inclusive schools: parental perspectives on family-school partnerships.

Giovacco-Johnson, T. (2009). Portraits of partnership: the hopes and dreams project. Early Childhood Education Journal, 37, 127-135. doi:10.1007/s 10643-009-0332-1

Goddard, R. D., Tschannen-Moran, M. y Hoy, W. K. (2001). A multilevel examination of the distribution and effects of teacher trust in students and parents in urban elementary schools. The Elementary School Journal, 3-17. doi:10.1086/499690

Govier, T. (1997). Social trust and human communities. Montreal: McGill-Queen’s Press.

Gurr, D., Drysdale, L. y Mulford, B. (2005). Successful principal leadership: australian case studies. Journal of Education Administration 43(6), 539-551.

Haines, S. J., McCart, A. y Turnbull, A. P. (2013). Family engagement within early childhood Response to Intervention. En V. Buysse y E. Peisner-Feinberg (Eds.), Handbook on Response to Intervention (RTI) in early childhood (pp. 313-324). Nueva York: Brookes.

Habegger, S. (2008). The principal's role in successful schools: creating a positive school culture. Principal, 88(1), 42-46.

Hill, N. E. y Taylor, L. C. (2004). Parental school involvement and children's academic achievement: Pragmatics and issues. Current Directions in Psychological Science, 13, 161-164. doi:10.1111/j.0963-7214.2004.00298.x

Hoy, W. K. y Tarter, C. J. (1997). The road to open and healthy schools: a handbook for change. Thousand Oaks, CA: Corwin Press.

Leithwood, K., Patten, S. y Jantzi, D. (2010). Testing a conception of how school leadership influences student learning. Education Administration 46(5) 671-706. doi: $10.1177 / 0013161 \times 10377347$

Martinez, D. C., Conroy, J. W. y Cerreto, M. C. (2012). Parent involvement in the transition process of children with intellectual disabilities: the influence of inclusion on parent desires and expectations for postsecondary education. Journal of Policy and Practice in Intellectual Disabilities, 9(4), 279-288. doi:10.1111/jppi.12000

Maxwell, J. A. (2005). Qualitative research design: An interactive approach. Thousand Oaks, CA: Sage.

McKinney, C. L., Labat, M. B. y Labat, C. A. (2015). Traits possessed by principals who transform school culture in national blue ribbon schools. Academy of Educational Leadership, 19, 152-166.

Peterson, K. D. y Deal, T. E. (2002). The shaping school culture fieldbook. San Fransisco, CA: Jossey-Bass 
Sailor, W. (2009). Making RTI work: How smart schools are reforming education through schoolwide response-to-intervention. San Francisco, CA: Jossey-Bass.

Sailor, W. (2014). Advances in schoolwide inclusive school reform. Remedial and Special Education, 36(2), 94-99. doi:10.1177/0741932514555021

Sailor, W., McCart, A., McSheehan, M., Mitchiner, M. y Quirk, C. (2014). SWIFT intensive technical assistance process. Lawrence, KS: SWIFT Center.

Sermier Dessemontet, R. y Bless, G. R. (2013). The impact of including children with intellectual disability in general education classrooms on the academic achievement of their low-, average-, and high-achieving peers. Journal of Intellectual and Developmental Disability, 38(1), 23-30. doi:10.3109/13668250.2012.757589

Shogren, K., McCart, A., Lyon, K. y Sailor, W. (2015) All means all: building knowledge for inclusive schoolwide transformation. Research \& Practice for Persons with Severe Disabilities, 4O(3), 173-191. doi:10.1177/1540796915586191

Sweetland, S. R. y Hoy, W. K. (2000). School characteristics and educational outcomes: toward an organizational model of student achievement in middle schools. Educational Administration Quarterly, 36(5), 703-729. doi:10.1177/00131610021969173

Waldron, N. L. y McLeskey, J. (2010). Establishing a collaborative school culture through comprehensive school reform. Journal of Educational and Psychological Consultation, 20(1), 58-74. doi:10.1080/10474410903535364

TASH (2012). Dispelling the myths of inclusion education. Recuperado de http://tash.org/wpcontent/uploads/2015/04/Myths-of-IE.pdf

Tschannen-Moran, M. (2001). Collaboration and the need for trust. Journal of Educational Administration, 39, 308-331. doi:10.1108/EUM0000000005493

Tschannen-Moran, M. (2014). Trust matters: Leadership for successful schools. San Francisco, CA: Jossey-Bass.

\section{Breve CV de las autoras}

\section{Grace L. Francis}

Assistant Professor of Special Education at George Mason University. Her research interests include family-professional partnership policies and practices and post-school outcomes that result in a high quality of life for individuals with significant support needs. Email: gfranci4@gmu.edu

\section{Judith M. S. Gross}

Assistant Research Professor at the Beach Center on Disability at the University of Kansas and a member of the SWIFT Family and Community Engagement Team, which provides technical assistance to SWIFT partner schools. Dr. Gross is also the principal investigator of the Assessing Family Employment Awareness Training research project which provides training and technical assistance to families that raises expectations for and knowledge of competitive employment for individuals with disabilities. Her research 
interests include family-disability policy, competitive employment, participant direction of supports and services, and community access for individuals with disabilities. Email: jgross@ku.edu

\section{Martha Blue-Banning}

Qualitative researcher on the School-Wide Integrated Framework for Transformation (SWIFT) Project at the Beach Center on Disability at the University of Kansas. Dr. Blue-Banning is the co-director of the SWIFT Family-Community-School Engagement team. Her primary research focus has been on parent-professional partnerships and the transition of adolescents and young adults with disabilities to an inclusive life in the community. Her other research interests include cognitive coping and participant direction of Medicaid Waivers. Email:mnn@ku.edu

\section{Shana J. Haines}

Assistant Professor in the College of Education and Social Services at the University of Vermont. Dr. Haines' research interests include family and community partnerships, improving schools, effective teacher education, and refugee and former refugee education. Email: sjhaines@uvm.edu

\section{Ann P. Turnbull}

Distinguished Professor in the Department of Special Education and Co-director of the Beach Center on Disability at the University of Kansas. Dr. Turnbull's research interests focus in the areas of family-professional partnerships, family quality of life, and school/community inclusion. Email: turnbull@ku.edu 